\title{
A monochromatic laser-pulsed picosecond electron field-emitter
}

\author{
J.C.H.Spence, T. Vecchione*, U. Weierstall* and G. Hembree* \\ Department of Materials, Cambridge University, Cambridge, UK. CB2 3QZ. (Spence@asu.edu) \\ *Department of Physics, Arizona State University, Tempe, Az. 85287-1504.
}

In order to obtain time-resolved diffraction patterns from single domains, a pulsed electron source is needed which may be focused to nanometer dimensions [1]. The total intensity within a focused spot from a field-emission source exceeds that from a conventional source once the spot size falls below about 100nm. The use of photoemission-stimulated field emission (photo-field emission) is ideally suited for this purpose. Previous systems have used a fast laser, focused onto a tungsten field-emission tip, to trigger field-emission [2]. The tip is held at the threshold of field emission. Light pulses excite photo-electrons from below the Fermi level across the surface potential barrier, lowered by the extraction potential and small tip radius. Quantum yields exceeding unity are normally obtained, and peak currents of up to 2 Amps have been reported using $20 \mathrm{~ns}$ pulses from a single tip [3]. (Arrays of these have been proposed for free electron lasers and linear accelerators). Because of the differences in scale between the laser focus and the emitting area of the tip, very high laser power is needed for these tips. In our design, shown the figure, light pulses are led to the tip via a fiber-optic, whose sharpened end acts as the field emission source. The tip is coated with GaAs, from which field emission has recently been reported [4]. A small $1 \mathrm{~mW}$ diode laser provides sufficient power to initiate photo-field emission. In previous work with a similar design (using a TiC coated tip), 100ps pulses were produced [5]. By using GaAs we plan to obtain monochromatic emission. A channel-plate is used to give a field-emission image of the tip. This develops previous NSF-funded research at ASU on field-emission nanotips and their increased brightness [6].

Estimates of cold field-emission brightness give $6 \times 10^{9} \mathrm{~A} / \mathrm{cm}^{2} / \mathrm{sr}$ (or $1.3 \times 10^{29}$ particles / sec/ $\mathrm{cm}^{2} / \mathrm{sr} / \mathrm{eV}$ ), and a typical cold field-emitter in continuous running produces about 20 electrons per picosecond at the tip. Assuming a $2 \mathrm{~nm}$ source diameter, an emission half-angle of 18 degrees (1/e intensity, 0.31 sterad) we expect 1500 electrons in an 80 ps pulse, to be focused by magnetic lenses into a small probe. This corresponds to a current of $20 \mathrm{pA}$ at a repetition rate of $100 \mathrm{kHz}$, which is comparable with the currents used to obtain diffraction patterns from radiation-sensitive materials. The exposure time is $\mathrm{T}=\mathrm{T}(\mathrm{DC})^{*} \mathrm{~T}(\mathrm{Rep}) / \mathrm{t}$, where $\mathrm{T}(\mathrm{DC})$ is the exposure time needed with continuous illumination, $\mathrm{T}(\mathrm{Rep})$ is the repetition period and $\mathrm{t}$ is the pulse duration. (Recent continuous Ti:Sapphire laser-driven system with a silver photocathode [7] have demonstrate 700 femtosecond single-shot transmission electron diffraction at $60 \mathrm{kV}$ with about 4000 electrons and a 40 micron probe). While insufficient for imaging at high magnification, the "concentration" of energy in Bragg spot diffraction patterns will provide useful time-resolved information, and diffuse scattering during a phase transformation can also be recorded. With long recording times, this current is also sufficient to allow the recording of microdiffraction patterns from repetitive processes. Improvements on this performance can be expected from aberration-corrected lenses, which provide larger numerical aperture. The quantum limit to electron source brightness is set by the requirement for no more that one electron (fermion) per state in phase space (unlike bosons such as photons). Measured degeneracies [8] are about $10^{-5}$ rather than this value of unity mainly due to Coulomb repulsion [9]. Transverse trajectory displacements enlarge the virtual source size (reducing brightness), while longitudinal displacements (Boersch effect, [10]) broaden the energy spread. A high extraction field therefore reduces coulomb repulsion and time broadening of pulses [10]. Since the chamber also forms a point-projection electron microscope [11], it will be possible to investigate the beneficial effects of short pulses on radiation damage [12].

For a semiconductor photo-field-emitter for which the laser beam energy $\mathrm{E}_{\mathrm{L}}$ exceeds the bandgap $\mathrm{E}_{\mathrm{g}}$, electrons are promoted by the photoelectric effect across the band-gap into empty conduction band states, from which they tunnel into vacuum through the vacuum barrier, which is lowered by the external field at the tip. The lowest emission energy therefore occurs at the bottom of the 
conduction band, while the highest occurs at an energy $\mathrm{E}_{\mathrm{L}}$ above the top of the filled valence band (for intrinsic material). The electron beam emission energy width is therefore approximately $\mathrm{E}_{\mathrm{L}}-\mathrm{E}_{\mathrm{g}}$, and this may be selected at will by choice of laser diode energy. Band-bending at surfaces and the variation in the width of the tunneling barrier across the beam energy distribution will affect this slightly. Our apparatus therefore includes a low-energy electron spectrometer for analysis of the emission energy distribution. We are considering both GaAs coating of glass tips, and solid cones of GaAs bonded to the end of a fiber. Calculations of charge-carrier generation and diffusion are in progress. The absorption length of the laser light is small for above-band-gap light, however the diffusion length of electrons can be several microns. Previous work on this system [4] suggests that electron diffusion near the GaAs surface accounts for charge transport to the emitting area at the tip. In this work field and photofield emission were compared, and increases of $10^{4}$ in current were reported under illumination at $77 \mathrm{~K}$ and optimum biasing, with quantum yields in excess of unity.

In summary, our research is aimed at fabricating a high-brightness, picosecond, monochromatic, spatially coherent photo-field emitter whose energy spread may be reduced to millivolt levels by choice of laser wavelength. Polarized electron emission is a future possibility.

\section{References}

[7] J. Cao et al, Appl Phys. Letts. 83, (2003) .1044-1046

[4] V.D.Kalganov et al J. Phys. Cond. Matt. 15, (2003) p. 5171

[1] "Electron Microdiffraction", J. Spence, and J. Zuo, Plenum Press, New York, (1992).

[3] M. Boussoukaya et al Nucl. Instr. Meth. A279, (1989) 405-409.

[2] C. Garcia and C.A. Brau, Nucl. Instr. Meth. A483, (2001) 273-276.

[6] W. Qian, M. R. Scheinfein, and J. Spence, J. Appl Phys. 73, (1993) 7041-7045 .

[8] J. Spence, W. Qian, and M. P. Silverman, J. Vac Sci. A12,(1994) 542-547 .

[9] A. Veen, C. Hagen, J. Barth, P. Kruit, J. Vac Sci Tech. B19, (2001) 2038-2044.

[10] H. Rose and R. Spehr, Adv Elec and Electr Phys. Supp 13C (1983).

[5] M. Schmidt “A pulsed field-emitter". PhD Thesis Darmstadt University, Germany.

[11] U. Weierstall, J. Spence, M. Stevens, K. Downing, Micron 30, (1999) 335-338 .

[12] R. Neutze et al. Nature 406, (2000) 752-757. Thanks to EPSRC and NSF.

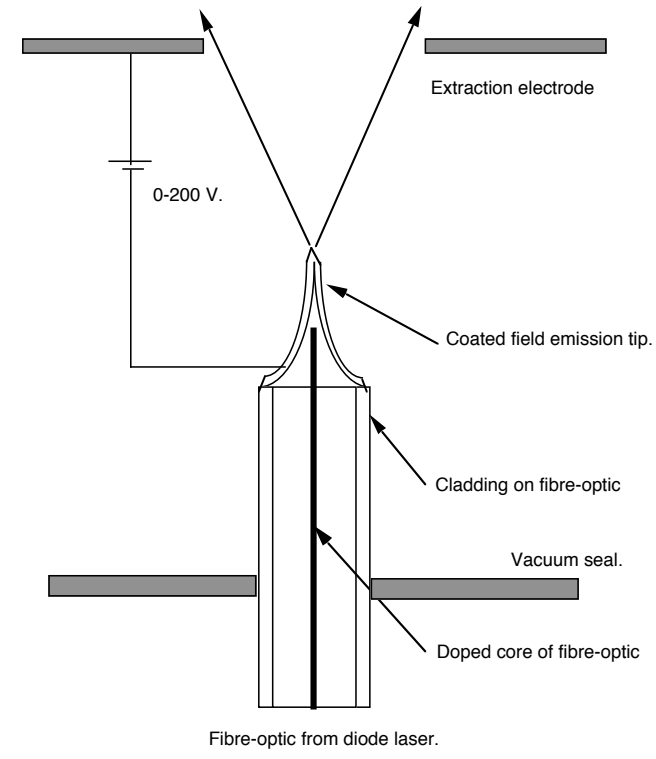

Figure 1. The pulsed photo-field-emission nanotip system. Light is guided to the GaAs coated tip by the fiber-optic. Tips are etched in HF and coated with gallium arsenide The distance between tip and extraction electrode (10 micron diameter) can be varied by moving the tip with an inch worm. 